\title{
Controllability of affine right-invariant systems on solvable Lie groups
}

\author{
Yuri L. Sachkov ${ }^{\dagger}$ \\ Program Systems Institute, Russian Academy of Sciences, 152140 Pereslavl-Zalessky, Russia \\ E-Mail: sachkovesys.botik.ru
}

The aim of this paper is to present some recent results on controllability of right-invariant systems on Lie groups. From the Lie-theoretical point of view, we study conditions under which subsemigroups generated by half-planes in the Lie algebra of a Lie group coincide with the whole Lie group.

Keywords: controllability, right-invariant system, Lie group

\section{Introduction}

First we recall definitions and state our problem.

Let $G$ be a real connected Lie group, $L$ be its Lie algebra (i.e. the set of all right-invariant vector fields on $G$ ). For any $A, B_{1}, \ldots, B_{m} \in L$ we consider the corresponding affine right-invariant system

$$
\Gamma=\left\{A+\sum_{i=1}^{m} u_{i} B_{i} \mid \forall i u_{i} \in \mathbb{R}\right\}
$$

The attainable set $\mathbf{A}$ for the system $\Gamma$ is a subsemigroup of $G$ generated by one-parameter semigroups $\{\exp (t X) \mid X \in \Gamma, t \geq 0\}$ The system $\Gamma$ is called controllable if $\mathbf{A}=G$.

We seek conditions which characterize controllability of the system $\Gamma$ in terms of the Lie group $G$, Lie algebra $L$ and vector fields $A, B_{1}, \ldots, B_{m}$.

Invariant control systems on Lie groups were first considered in 1972 by Brockett [1] and Jurdjevic and Sussmann [2] for the following reasons:

- Some mechanical systems evolve naturally on Lie groups, e.g. the group $\mathrm{SO}(3)$ is a state space for a rigid body rotating around its centre of mass.

\footnotetext{
${ }^{\dagger}$ The author thanks Professor Gérard Jacob and the organizers of the workshop 'Lie Computations' for financial support to enable participation in the workshop. This work was partially supported by the Russian Foundation for Fundamental Research, projects No. 96-01-00805 and No. 97-1-1a/22. The author is a recipient of the Russian State Scientific Stipend for 1997.
} 
- Invariant systems generate control systems on the homogeneous spaces of Lie groups. If $\Gamma$ is an invariant system on a Lie group $G, G / H$ is a homogeneous space of $G$, and $\pi: G \rightarrow G / H$ is a natural projection, then we can define $\pi_{*}(\Gamma)$ - a control system on $G / H$ which is a projection of $\Gamma$. It is well known that controllability of $\Gamma$ on $G$ implies controllability of $\pi_{*}(\Gamma)$ on $G / H$. For example, if $G \subset \operatorname{GL}(n)$ is a Lie group acting transitively on $\mathbb{R}^{n} \backslash\{0\}$, then we can take $G / H=\mathbb{R}^{n} \backslash\{0\}$, and $\pi_{*}(\Gamma)$ is a bilinear system

$$
\dot{x}=A x+\sum_{i=1}^{m} u_{i} B_{i} x, \quad u_{i} \in \mathbb{R}, x \in \mathbb{R}^{n} \backslash\{0\}
$$

The structure of this paper is as follows. In Sect. 2 we recall some known results about invariant systems on Lie groups. In Sect. 3 we give a controllability test for the hypersurface invariant systems. And in Sects. 4 and 5 we present and discuss controllability results for invariant systems on solvable Lie groups. The complete proofs of the theorems of Sects. $3-5$ are given elsewhere $[3,4]$.

\section{Known Results}

\subsection{Rank Condition}

The basic properties of attainable sets for invariant systems were studied by Jurdjevic and Sussmann [2]. Here we recall some of them.

The system $\Gamma$ is said to satisfy the rank controllability condition if the Lie algebra generated by the system coincides with $L$ :

$$
\operatorname{Lie}(\Gamma)=\operatorname{Lie}\left(A, B_{1}, \ldots, B_{m}\right)=L
$$

The important role of this condition is established by the following theorem.

Theorem 2.1 (Theorem 7.1 [2]) The rank controllability condition is necessary for controllability of $\Gamma$. If $G$ is compact or if $\Gamma$ is homogeneous (i.e. $A=0$ ), this condition is also sufficient.

Unfortunately, in the general case the rank condition is not sufficient for controllability, and substantial work was performed to obtain controllability conditions for invariant systems on various particular classes of Lie groups.

\subsection{Lie Saturation}

In 1981, Jurdjevic and Kupka [5] proposed an efficient method of investigation of controllability, and applied it to invariant systems on simple and semi-simple Lie groups. This method is based on the technique of Lie saturation, which may be stated as follows.

Given an invariant system $\Gamma$, there is defined a new system $\operatorname{LS}(\Gamma)$ called Lie saturation of $\Gamma$ :

$$
\operatorname{LS}(\Gamma)=\operatorname{Lie}(\Gamma) \cap\{X \in L \mid \exp (t X) \in \overline{\mathbf{A}} \forall t \geq 0\}
$$

A sufficient controllability condition in terms of Lie saturation was given:

Theorem 2.2 (Proposition $6[5]$ ) If $\mathrm{LS}(\Gamma)=L$, then $\Gamma$ is controllable. 
There is no algorithm to construct $\operatorname{LS}(\Gamma)$, but several nice properties are established which make it possible to extend $\Gamma$ and construct a chain of lower estimates of $\operatorname{LS}(\Gamma)$ :

$$
\Gamma \subset \Gamma_{1} \subset \ldots \subset \Gamma_{N} \subset \ldots \subset \operatorname{LS}(\Gamma) \subset L
$$

These properties are as follows (Proposition 5 [5]):

- $\operatorname{LS}(\Gamma)$ is a convex closed cone in $L$;

- if $\pm X, \pm Y \in \operatorname{LS}(\Gamma)$, then $\pm[X, Y] \in \operatorname{LS}(\Gamma)$;

- if $\pm X, Y \in \operatorname{LS}(\Gamma)$, then $\exp (s \operatorname{ad} X) Y \in \operatorname{LS}(\Gamma)$ for all $s \in \mathbb{R}$;

- if $X \in \operatorname{LS}(\Gamma)$ and one-parameter subgroup $\{\exp (t X) \mid t \in \mathbb{R}\}$ is periodic, then $-X \in \operatorname{LS}(\Gamma)$.

Thus, if we manage to construct an extension $\Gamma_{N}$ of the system $\Gamma$ such that $\Gamma_{N}=L$, then we can conclude that $\operatorname{LS}(\Gamma)=L$, and $\Gamma$ is controllable.

Extensive controllability conditions for invariant systems on simple and semi-simple Lie groups were obtained with the help of the Lie saturation technique by Jurdievic and Kupka [5]. and in subsequent papers by Gauthier and Bornard [6], Gauthier et al. [7] and Assoudi and Gauthier [8, 9].

\subsection{Systems on Nilpotent Groups}

There were almost no results on invariant systems on Lie groups different from simple and semi-simple groups. One of the possible reasons is that there is little known about the structure of Lie algebras except for simple and semi-simple algebras.

Ayala and Vergara [10] introduced the notion of a symplectic vector, and gave a necessary controllability condition in terms of this notion. Ayala Bravo [11] applied this condition and obtained a complete characterization of controllability for affine systems on nilpotent simply connected Lie groups:

Theorem 2.3 (Theorem 3.6 [11], Let $G$ be a nilpotent simply connected Lie group. Then the system $\Gamma$ is controllable iff Lie $\left(B_{1}, \ldots, B_{m}\right)=L$.

\section{Hypersurface Systems}

The main aim of this paper is to study invariant systems on solvable Lie groups. However, we first consider a particular class of invariant systems - hypersurface systems. Controllability conditions for this class are given in Theorem 3.1; they are important in themselves, and will be applied to obtain necessary controllability conditions for systems on solvable Lie groups (Corollary 3.1).

A nonlinear control system on a smooth manifold $M$

$$
\Sigma: \quad \dot{x}=A(x)+\sum_{i=1}^{m} u_{i} B_{i}(x), u_{i} \in \mathbb{R}, x \in M
$$

is called a hypersurface if we have

$$
\operatorname{dim} \operatorname{Lie}\left(B_{1}, \ldots, B_{m}\right)(x)=\operatorname{dim} M-1 \quad \forall x \in M
$$


Roughly speaking, hypersurface systems have $(n-1)$ independent inputs and $n$-dimensional state space. Such systems received a substantial attention (see, for example, Bacciotti and Stefani [12], Hunt 「13. 14] and Basto Goncalves [15]). The main idea is that for a hypersurface system $\Sigma$ the state space $M$ is stratified into codimension one foliation by the maximum integral manifolds of the distribution Lie $\left(B_{1}, \ldots, B_{m}\right)(x)$.

Now let us consider a hypersurface invariant system $\Gamma$, i.e. we suppose that the Lie algebra $L_{0}=$ Lie $\left(B_{1}, \ldots, B_{m}\right)$ is a codimension one subalgebra of $L$. Denote by $G_{0}$ the connected Lie subgroup of $G$ corresponding to $L_{0}$. It follows from the right-invariance of the fields $A(x)$ and $B_{1}(x), \ldots, B_{m}(x)$ that the field $A(x)$ does not intersect $G_{0}$ in both directions. This idea makes it possible to obtain a controllability test for hypersurface invariant systems:

Theorem 3.1 Suppose that $\operatorname{dim} L_{0}=\operatorname{dim} L-1$.

1. If $G_{0}$ is closed in $G$, then $\Gamma$ is controllable iff $A \notin L_{0}$ and $G / G_{0}=S^{1}$.

2. If $G_{0}$ is not closed in $G$, then $\Gamma$ is controllable iff $A \notin L_{0}$.

Remark. This theorem generalizes an analngnus result ohtained by Ayala Bravo [11] under the additional assumption that $L_{0}$ is an ideal of $i$, (Proposition 3.3 [11]).

It easily follows from the above theorem that hypersurface systems cannot be controllable on simply connected Lie groups. The reason is that a simply connected Lie group is separated into two disjoint components by the codimension one layer $G_{0}$. If we take any invariant system $\Gamma$ on a simply connected Lie group and manage to extend it to a hypersurface system $\Gamma_{1} \supset \Gamma$, then $\Gamma$ is not controllable.

Corollary 3.1 Let $G$ be simply connected. Suppose that there exists a codimension one subalgebra of $L$ containing $L_{0}$. Then the system $\Gamma$ is not controllable.

The idea of search for a hypersurface extension is a key one in the subsequent necessary conditions for systems on solvable simply connected Lie groups.

\section{Completely Solvable Lie Groups}

First we consider invariant systems on a subclass of solvable Lie groups appropriate for generalization of the test of Ayala Bravo [11] for nilpotent groups (Theorem 2.3). The sense of this test is that the drift term $A$ cannoi heip io coniroi the system $\Gamma=\left\{A+\sum_{i=1}^{m} u_{i} B_{i}\right\}$, i.e. the system $\Gamma$ is controllable if and only if the system without drift term $\Gamma_{0}=\left\{\sum_{i=1}^{m} u_{i} B_{i}\right\}$ is controllable. The 'if' part is obvious: we can take sufficiently great $u_{i}$ for the system $\Gamma$ and move almost along the trajectories of the system $\Gamma_{0}$. So the nontrivial part of the proof is the 'only if' part.

A (real) Lie algebra $L$ is called completely solvable if for any $X \in L$ the adjoint operator ad $X: L \rightarrow L$ has only real eigenvalues. Any completely solvable Lie algebra is solvable. A Lie group with completely solvable Lie algebra is called completely solvable.

Theorem 4.1 Suppose that $G$ is completely solvable and simply connected. Then

$\Gamma$ is controllable on $G$ iff Lie $\left(B_{1}, \ldots, B_{m}\right)=L$ 
The main idea of the proof of this theorem is that in a completely solvable Lie algebra $L$ any proper subalgebra can be extended to a codimension one subalgebra of $L$. Hence, any affine system can be extended to a hypersurface system (which is noncontrollable by Corollary 3.1).

Example 1. Let T $(n)$ denote the Lie group of $n \times n$ triangular matrices with positive diagonal entries. $\mathrm{T}(n)$ is connected, simply connected, and completely solvable. So Theorem 4.1 applies to invariant systems on $\mathrm{T}(n)$ or any connected subgroups of $\mathrm{T}(n)$.

Remark. Consider the following increasing chain of classes of Lie groups:

$$
\text { Commutative } \subset \text { Nilpotent } \subset \text { Completely solvable } \subset \text { Solvable }
$$

Suppose that Lie group $G$ is connected and simply connected. It is obvious that the property (*) is satisfied for commutative $G$. Theorem 2.3 establishes that (*) holds for nilpotent $G$, and Theorem 4.1 states that $(*)$ is true for completely solvable $G$. The results of the following section (in particular, Example 2) imply that $(*)$ is not valid for general solvable $G$.

\section{General Solvable Lie Groups}

Now we restrict ourselves by the single-input systems

$$
\Gamma=\{A+u B \mid u \in \mathbb{R}\}
$$

as the case of general solvable Lie groups is much more complicated. It turns out that there exist strong necessary conditions for the system $\Gamma$ to be controllable on solvable simply connected Lie groups (see Theorem 5.1 below). And if these necessary conditions are satisfied, there exist generic sufficient conditions which guarantee controllability (Theorem 5.2).

\subsection{Notation}

To state the controllability results precisely we need some notation.

Derived subalgebra and the second derived subalgebra:

$$
L^{(1)}=[L, L], \quad L^{(2)}=\left[L^{(1)}, L^{(1)}\right]
$$

Complexifications of $L, L^{(1)}$, and $L^{(2)}$ :

$$
L_{c}=L \otimes \mathbb{C}, \quad L_{c}^{(1)}=L^{(1)} \otimes \mathbb{C}, \quad L_{c}^{(2)}=L^{(2)} \otimes \mathbb{C}
$$

Adjoint representations:

$$
\operatorname{ad}: L \rightarrow \operatorname{End}(L), \quad \operatorname{ad}_{c}: L_{c} \rightarrow \operatorname{End}\left(L_{c}\right)
$$

Spectrum of ad $B$ in $L^{(1)}$ :

$$
\operatorname{Sp}^{(1)}=\left\{a \in \mathbb{C} \mid \operatorname{Ker}\left(\left.\operatorname{ad}_{c} B\right|_{L_{c}^{(1)}}-a \mathrm{Id}\right) \neq\{0\}\right\}
$$

Spectrum of ad $B$ in $L^{(2)}$ :

$$
\operatorname{Sp}^{(2)}=\left\{a \in \mathbb{C} \mid \operatorname{Ker}\left(\left.\operatorname{ad}_{c} B\right|_{L_{c}^{(2)}}-a \mathrm{Id}\right) \neq\{0\}\right\}
$$




\subsection{Necessary Conditions}

The necessary controllability conditions are obtained with the help of Corollary 3.1. Solvable Lie algebras possess a great number of codimension one subalgebras, and it is often possible to extend the system $\Gamma$ to a hypersurface system and show noncontrollability of $\Gamma$.

Theorem 5.1 Suppose that $G$ is solvable and simply connected. Let the spectrum $\mathrm{Sp}^{(1)}$ be simple. If $\Gamma$ is controllable, then:

1. $\operatorname{dim} L^{(1)}=\operatorname{dim} L-1$;

2. $B \notin L^{(1)}$;

3. $\mathrm{Sp}^{(2)} \cap \mathbb{R}=\mathrm{Sp}^{(1)} \cap \mathbb{R}$.

\subsection{Sufficient Conditions}

We obtain sufficient controllabilty conditions with the help of the Lie saturation technique. To show that $\operatorname{LS}(\Gamma)=L$ we consider the eigenvectors of the operator ad $\left.B\right|_{L^{(1)}}$ and construct a chain of these eigenvectors which generate $L^{(1)}$ step-by-step.

Theorem 5.2 Suppose that $G$ is solvable. Let the spectrum $\mathrm{Sp}^{(1)}$ be simple and all necessary conditions of Theorem 5.1 be satisfied. Suppose also that:

1. vector $A$ has nonzero components in all eigenspaces of $\left.\mathrm{ad} B\right|_{L^{(1)}}$;

2. $\mathrm{Sp}^{(1)} \subset\{\operatorname{Re} z>0\}$ or $\mathrm{Sp}^{(1)} \subset\{\operatorname{Re} z<0\}$ or $\mathrm{Sp}^{(1)} \cap \mathbb{R}=\emptyset$.

Then the system $\Gamma$ is controllable.

Example 2. Let $G=\mathrm{E}(2)$ be the Euclidean group of motions of $\mathbb{R}^{2}$. $\mathrm{E}(2)$ is connected but not simply connected. It can be represented as a group of $3 \times 3$ matrices of the form

$$
\left(\begin{array}{ccc}
c_{11} & c_{12} & b_{1} \\
c_{21} & c_{22} & b_{2} \\
0 & 0 & 1
\end{array}\right), \quad C=\left(c_{i j}\right) \in \mathrm{SO}(2), \quad b=\left(\begin{array}{c}
b_{1} \\
b_{2}
\end{array}\right) \in \mathbb{R}^{2}
$$

where $C$ is the rotation matrix and $b$ is the translation vector. The corresponding matrix Lie algebra $L$ is spanned by the matrices $A_{1}=E_{13}, A_{2}=E_{23}$ and $A_{3}=E_{21}-E_{12}$. We have $L^{(1)}=\operatorname{span}\left(A_{1}, A_{2}\right)$ and $L^{(2)}=\{0\}$; therefore, $L$ is solvable.

Consider the system $\Gamma=\{A+u B \mid u \in \mathbb{R}\}$ on $\tilde{\mathrm{E}}(2)$ - the simply connected covering of $\mathrm{E}(2)$. A complete characterization of controllability of $\Gamma$ on $\tilde{\mathrm{E}}(2)$ with the help of the above results:

Proposition 5.1 The system $\Gamma$ is controllable on $\tilde{\mathrm{E}}(2)$ if and only if vectors $A, B$ are linearly independent and $B \notin \operatorname{span}\left(A_{1}, A_{2}\right)$.

Really, if $B \in \operatorname{span}\left(A_{1}, A_{2}\right)$, then the system $\Gamma$ can be extended to a hypersurface system

$$
\Gamma_{1}=\left\{A+v_{1} A_{1}+v_{2} A_{2} \mid v_{1}, v_{2} \in \mathbb{R}\right\}
$$

so $\Gamma$ is noncontrollable by Corollary 3.1. If the vectors $A$ and $B$ are linearly dependent, then Lie $(A, B) \neq$ $L$, the rank controllability condition is violated, and $\Gamma$ is noncontrollable by Theorem 2.1. Finally, if 
$B \notin \operatorname{span}\left(A_{1}, A_{2}\right)$ and vectors $A, B$ are linearly independent, then all conditions of Theorem 5.2 are satisfied, hence $\Gamma$ is controllable on $\tilde{\mathrm{E}}(2)$.

It would be interesting to compare controllability conditions for $\tilde{\mathrm{E}}(2)$ with the following conditions for $\mathrm{E}(2)$ (these conditions are easily obtained with the help of the controllability test given in Theorem $1[16]:$ :

Proposition 5.2 The system $\Gamma$ is controllable on $\mathrm{E}(2)$ if and only if vectors $A, B$ are linearly independent and $\operatorname{span}(A, B) \not \subset \operatorname{span}\left(A_{1}, A_{2}\right)$.

\section{Final Remarks}

In addition to the papers cited in this paper, there are very interesting results on controllability of invariant systems on nilpotent and solvable Lie groups obtained via Lie semigroups methods by Hilgert et al. [17] and Lawson [18]. These results cover general systems, but for affine ones they do not imply the detailed theory presented in this work. Recently, the author applied this theory to obtain a classification of controllable systems on small-dimensional simply connected solvable Lie groups [19, 20]

\section{References}

[1] Brockett, R. W. (1972). System theory on group manifolds and coset spaces. SIAM J. Control 10, 265-284.

[2] Jurdjevic, V. and Sussmann, H. (1972). Control Systems on Lie Groups, J. Differ. Equat. 12, $313-$ 329.

[3] Sachkov, Y. L. (1996). Controllability of hypersurface and solvable invariant sstems. J. Dynamical and Control Syst. 2, 55-67.

[4] Sachkov, Y. L. (1997). Controllability of right-invariant systems on solvable Lie groups. J. Dynamical and Control Systems 3 (to appear).

[5] Jurdjevic, V. and Kupka, I. (1981). Control systems on semi-simple Lie groups and their homogeneous spaces. Ann. Inst. Fourier, Grenoble 31(4), 151-179.

[6] Gauthier, J.-P. and Bornard, G. (1982). Contrôlabilité des systèmes bilinèaires. SIAM J. Control Optim. 20(3), 377-384.

[7] Gauthier, J.-P., Kupka, I. and Sallet, G. (1984). Controllability of right-invariant systems on real simple Lie groups. Systems \& Control Lett. 5, 187-190.

[8] El Assoudi, R. and Gauthier, J.-P. (1988). Controllability of right-invariant systems on real simple Lie groups of type $F_{4}, G_{2}, C_{n}$, and $B_{n}$. Math. Control Signals Syst. 1(1), 293-301.

[9] El Assoudi, R. and Gauthier, J.-P. (1989). Controllability of right-invariant systems on semi-simple Lie groups. New Trends in Nonlinear Control Theory Vol 122, pp. 54-64. Springer-Verlag.

[10] Ayala, V. and Vergara, L. (1991). Co-adjoint representation and controllability. Proyecciones 11(1), $37-48$. 
[11] Ayala Bravo, V. (1995). Controllability of nilpotent systems. In: Geometry in Nonlinear Control and Differential Inclusions, pp. 35-46. Banach Center Publications, Warszawa, Poland.

[12] Bacciotti, A. and Stefani, G. (1983). On the relationship between global and local controllability. Math. Systems Theory 16, 79-91.

[13] Hunt, R. (1980). Global controllability of nonlinear systems in two dimensions. Math. Systems Theory $13,361-376$.

[14] Hunt, R. (1982). $n$-Dimensional controllability with $(n-1)$ controls. IEEE Trans. Autom. Control 27, 113-117.

[15] Basto Goncalves, J. (1987). Controllability in codimension one. J. Differ. Equat. 68, 1-9.

[16] Bonnard, B., Jurdjevic, V., Kupka, I. and Sallet, G. (1981). Transitivity of families of invariant vector fields on semi-direct products of Lie groups. Trans. Amer. Math. Soc..

[17] Hilgert, J., Hofmann, K. H. and Lawson, J. D. (1985). Controllability of systems on a nilpotent Lie group. Beiträge zur Algebra und Geometrie 20, 185-190.

[18] Lawson, J. D. (1985). Maximal subsemigroups of Lie groups that are total Proc. Edinburgh Math. Soc. 30, 479-501.

[19] Sachkov, Y. L. (1997). Controllability of right-invariant systems on solvable Lie groups: A survey and some new results. Submitted.

[20] Sachkov, Y. L. (1997). Classification of controllability in small-dimensional solvable Lie algebras. In preparation. 